\title{
A629
}

\section{VARIATIONS GÉNETIQUES DE LA FERTILITÉ DES BREBIS MÉRINOS D'ARLES} \author{
avec la collaboration technique de D. LAJOUS \\ Station d'Amélioration génétique des Animaux, \\ Centre de Recherches de Toulouse, I. N.R.A., \\ B.P. 12, 31320 Castanet-Tolosan \\ * Station de Physiologie animale, \\ Centre de Recherches de Montpellier, I. N.R. A., \\ 34000 Montpellier
}

J. RAZUNGLES, G. RICORDEAU, L. TCHAMITCHIAN et M. PRUD'HON*

\section{RÉSUMÉ}

Par suite des contraintes de la transhumance, les brebis Mérinos d'Avles du Domaine du Merle sont soumises à une lutte principale de mai-début juin. Avant de participer à cette lutte avec les adultes, les antenaises sont saillies pour la première fois à un an, en octobre, et agnèlent en mars-avril.

Cette étude porte sur I 794 antenaises nées sur 7 campagnes de 1965 à I97I et issues de 99 béliers. Nous avons étudié le taux de fertilité à un an et à $\mathrm{r} 8$ mois, en distinguant les antenaises gestantes et vides.

Le taux de fertilité à un an est en moyenne de 65,8 p. Ioo et a une héritabilité de o,27. Le taux de fertilité en mai-juin des antenaises venant de mettre bas est en moyenne de 48,2 p. 100 et a une héritabilité de 0,17 . Le taux de fertilité en mai-juin des antenaises vides est de 68,3 p. roo, ce qui s'explique par un " effet-bélier " plus marqué que sur les antenaises précédemment gestantes.

Les différences entre années et entre descendances montrent qu'il est possible d'augmenter sérieusement les performances de reproduction en améliorant les conditions d'élevage et en sélectionnant les reproducteurs ayant un indice favorable.

\section{INTRODUCTION}

Le cycle de reproduction utilisé sur le troupeau ovin de race Mérinos d'Arles élevé au Domaine du Merle (Bouches du Rhône) permet d'étudier la fertilité des antenaises à un an en octobre et leur aptitude à revenir en œstrus et à être fécondées en mai-juin après avoir mis bas en mars-avril et allaité leur agneau pendant 70 jours.

I,e but de la présente étude est d'analyser la variabilité des performances de reproduction des brebis sur leurs deux premières luttes, et d'estimer les paramètres génétiques des caractères de fertilité. 
Cette estimation donnera lieu à un développement particulier à cause de la spécificité du caractère étudié. La même méthode pourrait être utilisée pour tous les caractères dits " tout ou rien " dont l'expression se traduit par une réponse binaire. En outre sera mis en évidence, dans un bref paragraphe, l'effet bélier en complément des études de PRUD'hon et Denoy (I969).

\section{MATÉRIEL E'T MÉTHODES}

\section{I. - Conduite du troupeau et matériel animal}

Depuis de nombreuses années, le troupeau ovin de race Mérinos d'Arles élevé au Domaine $d u$ Merle a été soumis à des contrôles systématiques tout en respectant un mode d'élevage traditionnel (PRUD'HON et al., I966; PRUD' HON, 1971). En ce qui concerne la reproduction, le système d'élevage se décompose en deux luttes : une lutte principale, du Io mai jusqu'au départ en transhumance vers le 20 juin, et une lutte de rattrapage en automne qui débute dès le retour de transhumance et dure un mois, du ro octobre au ro novembre. Jusqu'en 1964, les jeunes brebis étaient saillies pour la première fois vers I9 mois à la lutte de printemps. Pour améliorer la productivité du troupeau, PRUD'HON (197I-1972) a proposé de mettre les agnelles à la lutte de printemps dès l'âge de 8 mois, mais cette solution n'a pas donné de bons résultats.

Le système qui a été essayé dès I 965 , et mis en place en I966, consiste à mettre les antenaises pour la première fois à la lutte en octobre et à les remettre ensuite à la saillie lors de la lutte principale de mai-juin, en essayant d'utiliser pour induire l'activité sexuelle, le stimulus consécutif à l'introduction des béliers, c'est-à-dire "l'effet bélier " qui a été bien mis en évidence sur les adultes (Prud'hon et Denoy, I969).

Pour analyser la variabilité des performances de reproduction au cours des deux premières luttes, nous avons utilisé I 794 brebis nées pendant 7 années consécutives, de 1965 à 197 r, et issues de 99 béliers. Les données disponibles concernent le nombre d'antenaises présentes et fécondées en octobre, le nombre d'antenaises venues en œstrus (saillies) et fécondées à la lutte de mai-juin, le nombre de produits nés aux différents agnelages.

Les antenaises étant pratiquement toutes issues de mères âgées de plus de 2 ans, nous avons considéré dans cette étude que l'âge des mères ne pouvait avoir qu'une influence négligeable sur la fertilité de leur fille.

D'autre part, nous avons accouplé au hasard les mâles aux antenaises, ce qui a pour conséquence d'annuler en moyenne l'influence éventuelle de la variabilité des béliers mis en lutte et pères des agneaux.

\section{2. - Caractères considérés}

La fertilité étant définie comme le pourcentage de brebis fécondées sur roo brebis présentes à la lutte, nous posons :

$f_{1}$ : fertilité à la lutte d'octobre à I an;

$f_{2}$ : fertilité à la lutte du Io mai au 20 juin des antenaises ayant mis bas en mars-avril ;

$f_{\mathbf{2}}^{\prime}$ : pourcentage d'antenaises venues en œstrus du Io mai au 20 juin, parmi celles ayant mis bas en mars-avril ;

$f_{3}$ : fertilité à la lutte de mai-juin des antenaises non venues en œstrus ou non fécondées à leur Ire lutte d'octobre.

Pour chaque descendance, nous avons calculé un indice annuel en exprimant la fertilité de chaque lot de filles en écart à la moyenne annuelle du troupeau. Certains béliers ayant été utilisés sur plusieurs campagnes ( $\mathrm{I}$ à 6 ), nous avons ensuite calculé un indice cumulé en utilisant, pour chaque descendance, la moyenne pondérée des différences annuelles d'après le nombre de filles présentes à chaque lutte consédrée.i Le nombre d'indices calculés avec un mimimum de 8 filles présentes est de 63 indices $f_{1}$ et de 53 indices $f_{2}$.

Les venues en cestrus en mai-juin ont été régulièrement contrôlées, sauf en 1973.

La prolificité est définie comme le pourcentage de nés par rapport aux brebis ayant agnelé. Sa valeur est très faible jusqu'à deux ans : I, oI à la première mise bas en avril-mai et 1,07 à la deuxième mise bas en octobre. Par conséquent, nous ne tiendrons pas compte des éventuelles 
différences entre la prolificité des antenaises à un an et celle des antenaises fécondées pour la première fois à $19-20$ mois.

Tous les agneaux ont été soumis au contrôle de croissance I.T.O.V.I.C., en vue de l'estimation des poids à âge-type et de l'indice gain 30 - $\tilde{\text { T } 0}$ jours calculé sur les performances des agneaux mâles et femelles de chaque descendance. C'est cet index que nous avons retenu pour mettre en relation les aptitudes vitesse de croissance et fertilité des béliers.

\section{3. - - Méthodes d'estimation des paramètres génétiques}

\section{a) Héritabilité.}

Plusieurs méthodes estimant l'héritabilité de caractères tout ou rien, tels que la fertilité, peuvent être utilisées, et ont été étudiées par RAzungLEs (I975).

Nous avons employé ici la méthode de ROBERTSON et LERner (I949) à cause de sa grande simplicité d'utilisation.

Les paramètres pris en considération sont les suivants :

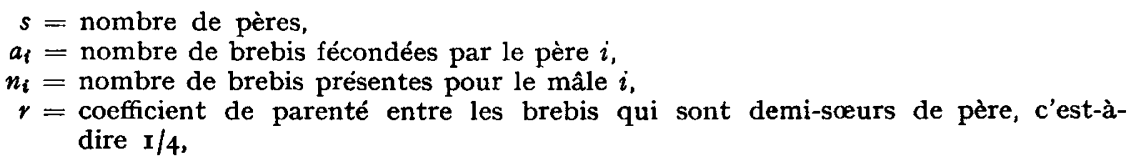

$$
\begin{aligned}
& n_{0}=\sum_{i=1}^{s} n_{i}-\frac{\sum_{i=1}^{s} n_{i}^{2}}{\sum_{i=1} n_{i}}-(s-1)
\end{aligned}
$$

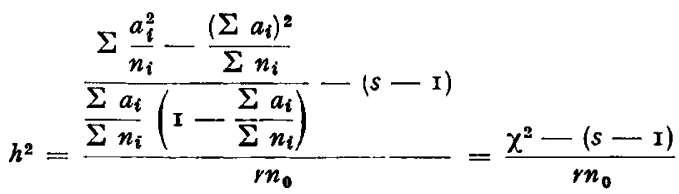

Toutes les sommations sont faites par rapport à l'indice $i$ qui varie de $\mathbf{I}$ à $s$.

Nous avons montré (RAzUNGLES, I975) que :

$$
\begin{aligned}
& \text { Variance } h^{2}=\frac{2 v}{r^{2} n^{2}(s-\mathrm{I})^{2}} \\
& \text { avec : } \quad v=(s-\mathrm{I})+\frac{\mathrm{I}}{n}\left(\mathrm{I}-\frac{\mathrm{I}}{s}\left(\mathrm{I}-\frac{\mathrm{I}}{n}\right)\right)
\end{aligned}
$$

$n$ étant le nombre moyen de filles de chaque mâle c'est-à-dire :

$$
n=\frac{\Sigma n_{i}}{s} .
$$

Pour $n$ assez grand on peut prendre $v$ égal à $s-\mathbf{r}$.

La moyenne globale $m$ des héritabilités pour l'expérience menée surplusieurs années a été obtenue en regroupant les résultats de la manière suivante :

Si pour l'année $j$ l'héritabilité est donnée par la formule :

$$
h_{j}^{2}=\frac{\chi_{i}^{2}-\left(s_{j}-I\right)}{r n_{0 j}},
$$


alors l'héritabilité globale sera :

$$
h^{2}=\frac{\sum_{j=1}^{m} \chi_{j}^{2}-\left(\sum_{j=1}^{m} s_{j}-\mathbf{I}\right)}{r \sum_{j=1}^{m} n_{0 j}}
$$

considérant pour cela que les $\chi_{j}^{2}$ sont indépendants, c'est-à-dire en fait, que l'homogénéité des mesures d'une année donnée est indépendante de l'homogénéité des mesures d'une autre année. La variance de cette héritabilité sera donnée par la même formule que pour chacune des années avec :

$$
n=\frac{\sum_{j=1}^{m} \sum_{i=1}^{s} n_{i j}}{\sum_{j=1}^{m} s_{j}}
$$

$n_{i j}$ étant le nombre de filles du ileme mâle, l'année $j$,

et :

$$
v=\sum_{j=1}^{m} s_{j}-\mathrm{I}+\frac{\mathrm{I}}{n}\left(\mathrm{I}-\frac{\mathrm{I}}{\sum_{i=1}^{m} s_{j}}\left(\mathrm{I}-\frac{\mathrm{I}}{n}\right)\right)
$$

En pratique on prendra toujours : $\quad v=\sum_{j=1}^{m} s_{j}-\mathbf{I}$.

b) Indices.

Pour chaque bélier, l'indice annuel est défini comme la fertilité de chaque lot de filles en écart à la moyenne annuelle du troupeau, et l'indice cumulé est la moyenne des indices annuels pondérés par l'inverse du nombre de filles présentes à chaque lutte considérée. Nous n'avons considéré que les mâles ayant au moins 8 filles présentes à la lutte.

Soient $n_{i j}$, le nombre de filles présentes pour le bélier $i$, l'année $j$ et parmi elles, $x_{i j}$ le nombre de celles qui ont mis bas, alors l'indice du bélier $i$ l'année $j$ sera :

et l'indice cumulé du bélier $i$ sera:

$$
\mathrm{B}_{i j}=\left(\frac{x_{i j}}{n_{i j}}-\frac{\sum_{i} x_{i j}}{\sum_{i} n_{i j}}\right)
$$

$$
\mathrm{B}_{i}=\frac{\sum_{j} n_{i j} \mathrm{~B}_{i j}}{\sum_{j} n_{i j}}
$$

\section{RÉSULTATS}

\section{I. - Variations annuelles et entre descendances}

\section{r. Fertilité à 1 an.}

De I966 à I972, le taux de fertilité à un an $\left(f_{1}\right)$ varie de 45,4 à 76,2 p. Ioo, autour d'une moyenne de 65,8 p. Ioo (tabl. I). 
TABLEAU I

Fertilité aux deux premières luttes ( 1 an et 18 mois) des brebis nées sur sept années consécutives

\begin{tabular}{|c|c|c|c|c|c|c|c|c|c|c|c|}
\hline \multirow{2}{*}{\multicolumn{2}{|c|}{$\begin{array}{c}\text { Année } \\
\text { de naissance }\end{array}$}} & \multirow{2}{*}{\multicolumn{2}{|c|}{$\begin{array}{c}\text { Fertilité } \\
\text { à } 1 \text { an } \\
\left(f_{1}\right)\end{array}$}} & \multicolumn{4}{|c|}{$\begin{array}{l}\text { Antenaises de } 18 \text { mois } \\
\text { fécondées à } 1 \text { an }\end{array}$} & \multirow{2}{*}{\multicolumn{2}{|c|}{$\begin{array}{c}\text { Fertilité } \\
\text { en mai pour } \\
\text { les antenaises } \\
\text { non fécondées } \\
\text { à } 1 \text { an }\left(f_{3}\right)\end{array}$}} & \multirow{2}{*}{\multicolumn{2}{|c|}{$\begin{array}{l}\text { Fertilité } \\
\text { globale }\end{array}$}} \\
\hline & & & & \multicolumn{2}{|c|}{$\begin{array}{c}\text { saillies en mai } \\
\left(f_{2}^{\prime}\right)\end{array}$} & \multicolumn{2}{|c|}{$\begin{array}{l}\text { fertilité en mai } \\
\qquad\left(f_{2}\right)\end{array}$} & & & & \\
\hline Octob & 1965 & 68,9 & (376) & 92,6 & (231) & 83,0 & (230) & 87,2 & (86) & 84,2 & (316) \\
\hline - & 1966 & 68,8 & (295) & 72,5 & (182) & 49,4 & (184) & 91,6 & (83) & 62,5 & (267) \\
\hline- & 1967 & 76,2 & (214) & 72,4 & (156) & 45,9 & (159) & 66,7 & (48) & 50,7 & (207) \\
\hline- & 1968 & 48,7 & (187) & 65,9 & (91) & 43,7 & (87) & 62,8 & (86) & 53,2 & (173) \\
\hline - & 1969 & 45,4 & (205) & 38,2 & (89) & 33,7 & (89) & 73,8 & (103) & 55,2 & (192) \\
\hline- & 1970 & 75,3 & (287) & 33,0 & (200) & 22,0 & $(200)$ & 32,8 & (67) & 24,7 & (267) \\
\hline- & 1971 & 67,8 & (230) & & & 40,7 & (135) & 50,8 & (65) & 44,0 & (200) \\
\hline \multicolumn{2}{|c|}{ Total } & \multicolumn{2}{|l|}{65,8} & \multicolumn{2}{|l|}{65,2} & \multicolumn{2}{|l|}{48,2} & \multicolumn{2}{|l|}{68,3} & \multicolumn{2}{|c|}{54,8} \\
\hline
\end{tabular}

TABLEAU 2

Distribution de fréquence des indices $f_{1}$ et $f_{\mathbf{2}}$

a) Suivant le niveau des indices

\begin{tabular}{|c|c|c|}
\hline Niveau & $\begin{array}{c}\text { Indice } f_{1} \\
(\%)\end{array}$ & $\begin{array}{c}\text { Indice } f_{2} \\
(\%)\end{array}$ \\
\hline $\begin{array}{rr}-38 \text { à }-25 \\
-25 \text { à }-15 \\
-15 \text { à }-5 \\
-\quad 5 \text { à } \\
5 \text { à } \\
15 \text { à } \\
25 \text { à } \\
25 \\
\end{array}$ & $\begin{array}{r}4,6 \\
10,6 \\
16,7 \\
33,3 \\
21,2 \\
10,6 \\
3,0\end{array}$ & $\begin{array}{r}1,9 \\
11,3 \\
22,6 \\
24,5 \\
30,2 \\
7,6 \\
1,9\end{array}$ \\
\hline
\end{tabular}

b) Suivant l'effectif de filles ayant servi au calcul des indices

\begin{tabular}{c|c|c}
\hline Effectif & $\begin{array}{c}\text { Indice } f_{\mathbf{1}} \\
(\%)\end{array}$ & $\begin{array}{c}\text { Indice } f_{\mathbf{2}} \\
(\%)\end{array}$ \\
\hline 8 à 12 & 19,7 & 28,3 \\
13 à 17 & 15,1 & 30,2 \\
18 à 22 & 18,2 & 17,0 \\
23 à 27 & 6,1 & 7,5 \\
28 à 32 & 15,1 & 13,2 \\
32 à 68 & 25,8 & 3,8 \\
\hline TotaL & 66 indices & 53 indices \\
\hline
\end{tabular}


Sur les 63 descændances possédant de 8 à 68 filles, l'indice cumulé varie de $-3^{8}$ à +32 p. I0o (tabl. 2).

\section{Fertilité à la lutte de mai-juin après une mise bas de mars-avril.}

Le taux de venues en ostrus $\left(f_{2}^{\prime}\right)$ varie de 92,6 en 1967 , à 33,0 p. Ioo en I972, autour d'une moyenne de 65,2 . Le taux de fertilité $f_{2}$ est inférieur et varie de 83,0 en I967 à 22,0 en I972, autour d'une moyenne de 48,2 p. Ioo.

L'intervalle mise bas- $\mathrm{I}^{\mathrm{re}}$ saillie, calculé uniquement sur les 4 premières années varie de 45 à 120 jours. Le taux de réussite (brebis fécondées en p. Ioo des brebis saillies) semble indépendant de cet intervalle (tabl. 3), aussi peut-on considérer que les fécondations ont lieu normalement à partir du $45^{\mathrm{e}}$ jour post-partum.

\section{TABLEAU 3}

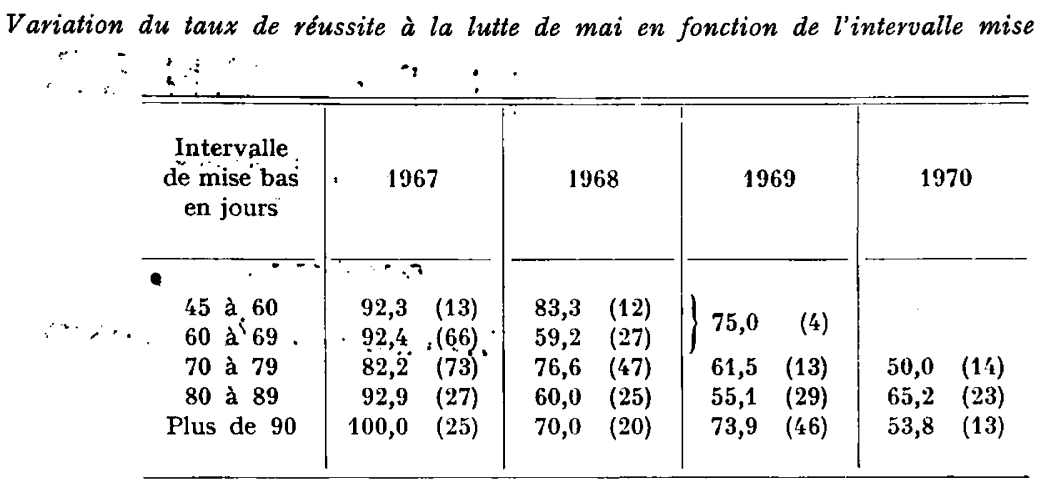

L'intervalle moyen de mise bas est de 74 et 76 jours en 1967 et 1968, de 90 et 87 jours en 1969 et 1970 .

Sur les 53 indices calculés sur 8 à 5 I filles, $f_{2}$ varie de -29 à +27 p. 100 (tabl. 2$)$; les limites de variation sont encore de -22 et +22 pour les $\mathrm{I} 6$ indices calculés sur plus de 20 filles.

\section{Fertilité en mai-juin pour les antenaises vides.}

L,e taux de fertilité $f_{3}$ varie de 9I,6 en I968 à 32,8 p. Ioo en I972, autour d'une moyenne de 68,3 p. Ioo (tabl. I). Il est dans tous les cas supérieur à $f_{2}(68,3$ contre 48,2 p. I00).

Finalement, le pourcentage d'antenaises fécondées en mai-juin par rapport à toutes celles présentes - vides ou gestantes à I an - est en moyenne de 54,8, mais les variations annuelles sont importantes : 84,2 en r $967,62,5$ en r 968 et 44,0 à 55 p. roo de 1969 à I973, mise à part la fertilité exceptionnellement faible observée en 1972 (24,7 p. roo). La fertilité globale des antenaises en mai-juin est donc, dans l'ensemble, nettement inférieure à celle des adultes au cours de la même lutte (90 p. Ioo ; PRUd'hon et Denoy, I969). 


\section{Observation de "l'effet bélier".}

En considérant les venues en œstrus de toutes les antenaises présentes à la lutte de mai-juin, on retrouve "l'effet bélier " signalé par PRUD'hoN et DENOY (I969). Cependant, pendant les ro premiers jours de lutte, le pourcentage de venues en œstrus est significativement différent pour les vides et les allaitantes (tabl. 4, fig. I). Si l'on exclut les observations de 1969 et 1970 (absence ou nombre insuffisant de béliers vasectomisés $x_{5}$ jours avant le début de la lutte), l'effet bélier est significatif uniquement pour les vides, avec 2 pics de venues en œstrus, I7 et 24 jours après l'introduction des béliers. Pour les allaitantes, on observe plusieurs pics (un premier au $I 7^{\mathrm{e}}$ jour, un second $\mathrm{du} 23$ au $26^{\mathrm{e}}$ jour et un $3^{\mathrm{e}}$ plus important au 33 et $34^{\mathrm{e}}$ jour) ce qui laisse supposer que la plupart de ces brebis ont eu, au préalable, au moins 2 ovulations silencieuses : en fait, les dates d'apparition du premier œestrus sur ces brebis sont peu différentes de celles observées sur les " tardonnières " par PRUD'HON et DENOY (I969).

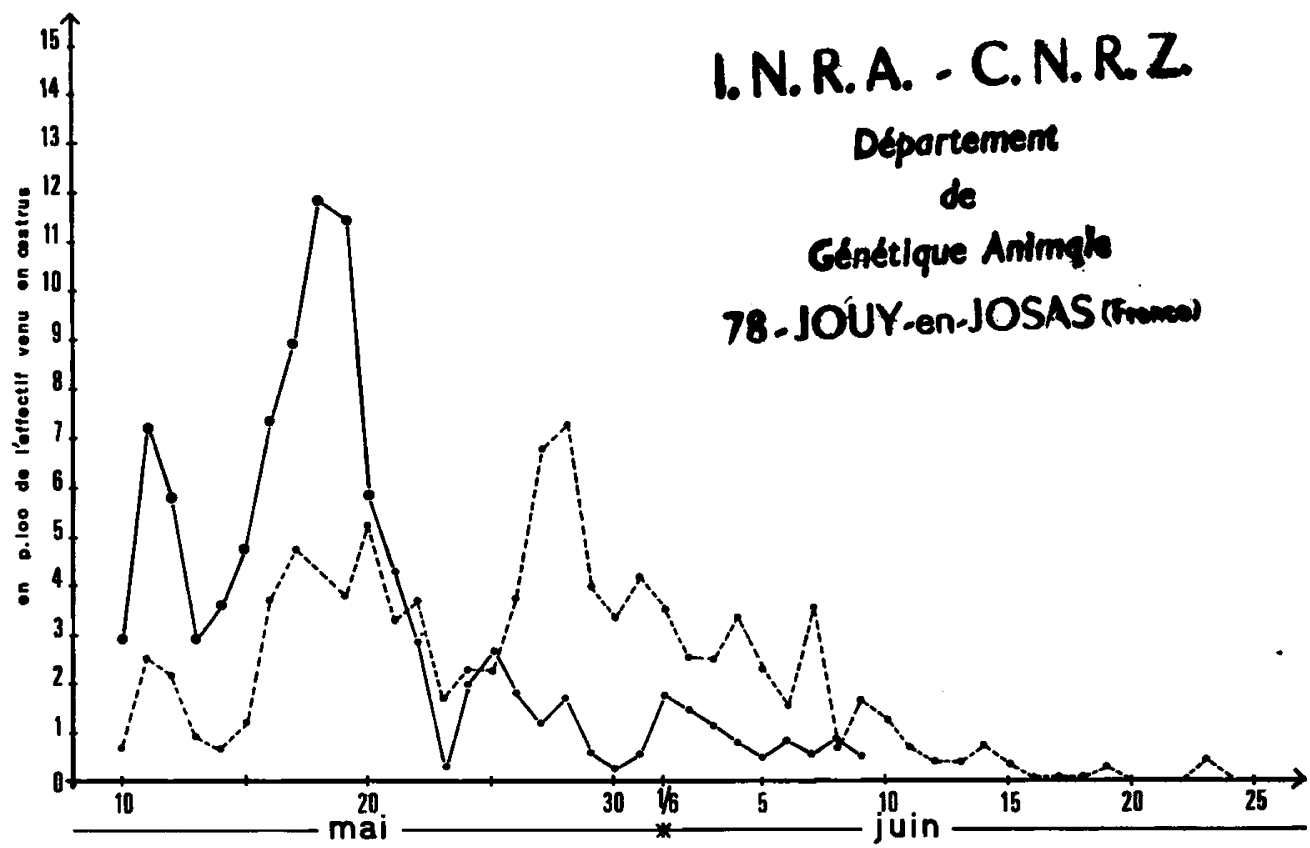

FIG. I. - Fréquence d'apparition des aestrus (saillies) en mai et juin pour les antenaises vides et allaitantes (5 années d'observations)

antenaises vides

5. Correspondance entre indices (tabl. 5).

La corrélation entre l'indice " gain 30-70 jours " et l'indice $f_{1}$ est positive, mais non significative $(+0, \mathrm{rg})$. La corrélation entre les indices $f_{1}$ et $f_{2}$ des 53 béliers possédant au moins 8 filles est également non significative $(+0,17)$. Cependant, lorsqu'on considère uniquement les 44 indices calculés avec 8 filles sur au moins 2 campagnes, la corrélation devient significative $(+0,3 I)$. 


\section{TABLEAU 4}

Importance de l'effet bélier à la lutte de mai sur les antenaises allaitantes et vides Brebis venues en œstrus les dix premiers jours de lutte en pourcentage des brebis saillies jusqu'au ro juin ( ${ }^{(1)}$

\begin{tabular}{|c|c|c|c|}
\hline Année & $\begin{array}{c}479 \\
\text { allaitantes }\end{array}$ & 339 vides & Observations \\
\hline 1967 & 42 & 80 & \multirow{8}{*}{$\begin{array}{l}\text { Absence ou insuffisance } \\
\text { de béliers vasectomisés } \\
15 \text { jours avant la lutte }\end{array}$} \\
\hline 1968 & 16 & 66 & \\
\hline 1971 & 20 & 65 & \\
\hline 1972 & 8 & 67 & \\
\hline 1973 & 12 & 70 & \\
\hline Moyenne (\%) & 24,8 & 67,0 & \\
\hline 1969 & 1 & 26 & \\
\hline 1970 & 6 & 32 & \\
\hline
\end{tabular}

(1) Dans le cas d'une distribution continue des brebis en œestrus sur un cycle, on peut espérer 58 p. 100 de venues en cestrus en 10 jours.

TABLEAU 5

Relations entre indices

\begin{tabular}{|c|c|c|c|c|c|c|}
\hline \multicolumn{2}{|c|}{ Variables } & \multirow{2}{*}{$\begin{array}{c}\text { Nombre } \\
\text { de } \\
\text { couples }\end{array}$} & \multirow{2}{*}{$r$} & \multirow{2}{*}{$b_{y, x}$} & \multirow{2}{*}{$\bar{x}$} & \multirow{2}{*}{$\bar{y}$} \\
\hline$x$ & $y$ & & & & & \\
\hline $\begin{array}{c}\text { Gain } \\
(30-70 \text { jours })\end{array}$ & $f_{1}$ & 34 & 0,19 & 0,79 & 1,0 & 1,7 \\
\hline$f_{1}$ & $f_{2}$ & $\begin{array}{l}53\left({ }^{1}\right) \\
44\left(^{2}\right)\end{array}$ & $\begin{array}{l}0,17 \\
0,31\end{array}$ & $\begin{array}{l}0,17 \\
0,32\end{array}$ & $\begin{array}{l}2,1 \\
1,0\end{array}$ & $\begin{array}{l}0,5 \\
0,7\end{array}$ \\
\hline$f_{2}^{\prime}$ & $f_{2}$ & 30 & 0,74 & 0,87 & $-0,4$ & 0 \\
\hline
\end{tabular}

(1) Indices $f_{1}$ et $f_{2}$ calculés avec un minimum de 8 filles.

(2) Indices $f_{1}$ et $f_{2}$ calculés avec un minimum de 8 filles sur 2 années. 
La corrélation entre les indices $f_{2}$ et $f_{2}^{\prime}$ est très significative, en dépit d'une observation qui se situe tout à fait en dehors de l'ellipse de dispersion.

\section{Estimation de l'héritabilité.}

Pour utiliser la méthode de ROBERTSON et LERNER (I949), nous avons effectué un calcul séparé par année. Cependant, certains mâles ayant des filles plusieurs années consécutives, les valeurs obtenues d'une année à l'autre ne sont pas totalement indépendantes.

L'héritabilité moyenne du taux de fertilité est de 0,25 pour $f_{1}$ et de 0,17 pour $f_{2}$ (tabl. 6).

TABLEAU 6

Héritabilités estimées à partir des descendances d'au moins 8 filles

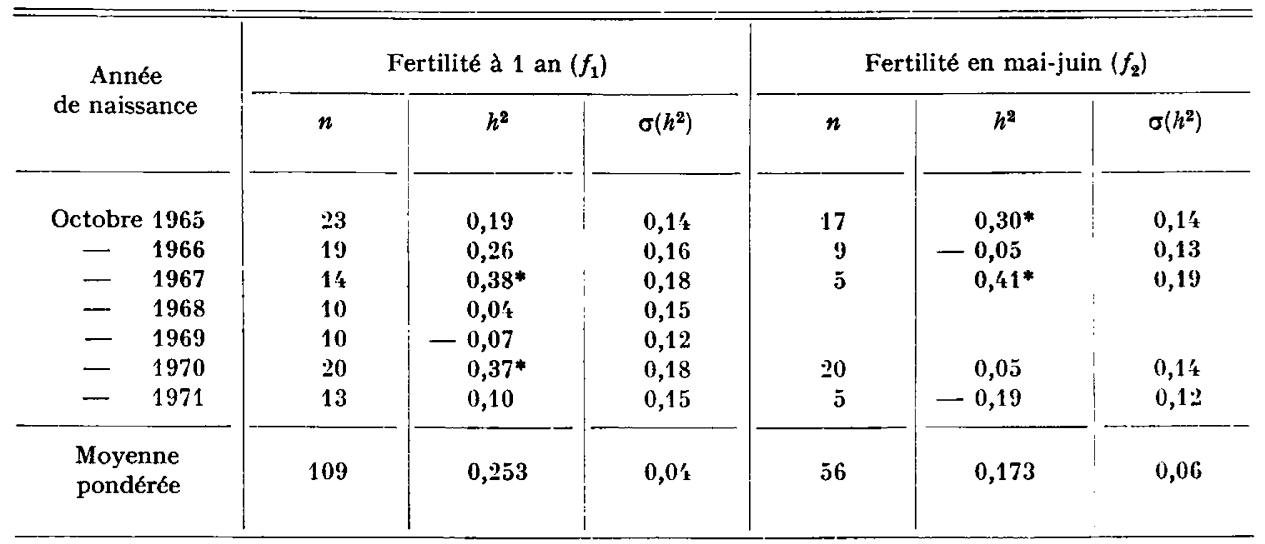

$n$ : nombre de descendances prises en considération chaque année.

\section{DISCUSSION ET CONCLUSIONS}

I. Les brebis Merinos d'Arles du troupeau du Merle ont une précocité sexuelle assez réduite puisque $65,8 \mathrm{p}$. Ioo seulement des antenaises sont fécondées à $\mathrm{I}$ an, à la lutte d'octobre dont la durée, il est vrai, ne couvre pas entièrement 2 cycles. Parmi celles restées vides après cette $\mathrm{I}^{\mathrm{re}}$ lutte, $68,3 \mathrm{p}$. Ioo sont fécondées à la $2^{\mathrm{e}}$ lutte de mai-juin. Aussi, globalement et sans tenir compte des réformes, sur roo antenaises présentes à I an, II seulement restent vides après les 2 premières luttes.

2. Sur les antenaises fécondées à $I$ an et qui mettent bas en mars-avril, 65,2 p. roo viennent en œstrus en mai-juin et 48,2 p. Ioo sont à nouveau fécondées. Au total donc, 3I,7 p. roo des antenaises sont capables d'être fécondées 2 fois de suite, en octobre à $\mathrm{I}$ an et en mai à I8 mois. Il s'agit là d'une aptitude assez remarquable de la race Mérinos d'Arles, compte tenu de la durée de l'ancestrus post-partum observée dans d'autres races (MAULÉon et DAUZIER, 1965). Des résultats au moins aussi bons ont été obtenus en Ile de France ou sur des Romanov 
et croisées Romanov $\times$ Berrichon $d u$ Cher (RICORDEAU et al., 1976), mais les conditions d'élevage et de reproduction sont très différentes. En race Ile de France, en particulier, l'absence des contraintes imposées par la transhumance permet aux éleveurs d'avancer la date de saillie des agnelles pour obtenir des agnelages en janvier-février et de prolonger la lutte de mai jusqu'en juin-juillet pour réaliser le maximum de fécondations. Dans le cas de la Mérinos d'Arles, si le système est en gros comparable, il faut rappeler que la $\mathbf{I}^{\mathrm{re}}$ lutte d'automne est tardive et courte - ce qui entraîne des agnelages de mars et avril — et que la lutte de printemps est limitée à 40 jours du fait du départ en transhumance.

3. Du point de vue génétique, l'héritabilité de la fertilité aux deux premières luttes est relativement faible mais significativement différente de zéro. L'estimation de l'héritabilité, chaque année, n'est pas suffisamment précise, mais un regroupement des estimations a permis de calculer une valeur globale en supposant que l'effet année est faible.

Cette supposition peut être faite pour la $f_{1}$ si l'on ne tient pas compte des années I 968 et I 969 . Pour la $f_{2}$ il est difficile de faire l'hypothèse d'homogénéité des estimations. Ainsi l'héritabilité globale calculée n'aura qu'une valeur tout au plus indicative, d'autant que le nombre de descendances contrôlées chaque année est en général très faible. L'héritabilité sur les $f_{2}^{\prime}$ et les $f_{3}$ n'ont pas été calculées à cause de cette dernière raison même.

4. Du point de vue pratique, les différences entre années mettent en évidence la nécessité d'augmenter le taux de fertilité à $\mathbf{I}$ an et vraisemblablement celui de la lutte de mai, en améliorant les conditions d'élevage. S'il n'est pas souhaitable de trop prolonger la durée de la première lutte d'octobre pour éviter les mises bas tardives l'année suivante, il faut améliorer l'état général des brebis afin d'obtenir notamment un meilleur poids à la lutte, puisque PRUD'HON et al. (I968) ont clairement montré l'influence du poids sur les performances de reproduction. Un gain de 15 à 20 points du taux de fertilité à $I$ an est possible. Il augmenterait d'autant les chances de réussite à la lutte principale de mai-juin, à condition d'utiliser un nombre suffisant de béliers vasectomisés $I_{5}$ jours avant le début des saillies.

Reçu pour publication en janvier 1976.

\section{REMERCIEMEN'TS}

Cette étude a été largement facilitée par la collaboration technique du C.T.G.R.E.F., Division techniques et économie des exploitations d'élevage bovin et ovin. Nous remercions l'Antenne Élevage Ovin de Montpellier et plus particulièrement M. Blanchemain.

\section{SUMMARY}

\section{GENETIC VARIATIONS IN FERTILITY OF MERINOS D'ARLES EWES}

Because transhumance Merinos d'Arles ewes at the Domaine du Merle have the principal breeding season in May-beginning of June. Before participating in this mating along with the adult sheep, the tegs are mated for the first time in October when they are one year old and they lamb in March-April. 
This report covers 1794 tegs, progeny of 99 rams, born in 7 years between 1965 and I97I. Fertility rate at one year and at 18 months is studied, distinguishing pregnant and barren tegs.

Mean fertility rate at one year is $65.8 \mathrm{p}$. 100 and has 0.27 heritability. Mean fertility rate in May-June of tegs which have recently lambed is $48.2 \mathrm{p}$. Ioo; heritability is o.17. Fertility rate in May-June of barren tegs is 68.3 p. 100 ; this is explained by a more marked " ram effect" than on the tegs which were pregnant.

Differences between years and between progeny show that reproductive performance can be considerably increased by improving breeding conditions and selecting breeding animals with a favorable index.

\section{RÉFÉRENCES BIBLIOGRAPHIQUES}

Mauléon P., Dauzier L., r965. Variations de la durée de l'ancestrus de lactation chez les brebis de race Ile de France. Ann. Biol. anim. Bioch. Biophys., 5, 13I-143.

Prud'hon M., I97I. Étude des paramètres influençant la fécondité des brebis et la mortalité des agneaux d'un troupeau de race Mérinos d'Arles. Thès. Univ. Sci. et Tech. Languedoc.

Prud'hon M., Denoy I., 1969. Effets de l'introduction des béliers vasectomisés dans le troupeau Mérinos d'Arles quinze jours avant le début de la lutte de printemps, sur l'apparition des œestrus, la fréquence des erreurs de détection des ruts et la fertilité des brebis. Ann. Zootech., 18, 95-106.

Prud'hon M., Denoy I., Dauzier L., Desvignes A., ig66. Étude des résultats de six années d'élevage des brebis Mérinos d'Arles du Domaine du Merle. I. Le contrôle des ruts et sa validité. Ann. Zootech., 15 I23-I33.

Prud'hon M., Denoy I., Desvignes A., Goussopoulos J., I968. Étude des résultats de six années d'élevage des brebis Mérinos $d^{\prime}$ Arles du Domaine du Merle. II. Relations entre l'âge, le poids, l'époque de lutte des brebis et les divers paramètres de la fécondité. Ann. Zootech., 17, 31-45.

Razungles J., 1975. Héritabilité des caractères discrets. Bibliographie critique. Ann. Génét. Sél.anim. (soumis à publication).

Ricordeau G., Tchamitchian L., Eychenne F., Razungles J., I976. Performances de reproduction des brebis Berrichon du Cher, Romanov et croisées. I. Activité sexuelle en début de saison et à contre saison. Ann. Génét. Sél. anim. (soumis à publication).

Robertson A., Lerner J. M., x949. The heritability of all-or-none trait. Genetics, 34, 395-4II. 